\title{
Correction to: Analysis of resistance genes of clinical Pannonibacter phragmitetus strain 31801 by complete genome sequencing
}

\author{
De-song Ming ${ }^{1} \cdot$ Qing-qing Chen ${ }^{1} \cdot$ Xiao-tin Chen $^{1}$
}

Published online: 27 September 2018

๑) Springer-Verlag GmbH Germany, part of Springer Nature 2018

\section{Correction to: \\ Archives of Microbiology (2018) 200:1101-1109 \\ https://doi.org/10.1007/s00203-018-1522-2}

Subsequent to publication it has been noticed that the below listed text parts, figures and tables of the above paper were taken from paper "Genomic insights of Pannonibacter phragmitetus strain 31801 isolated from a patient with a liver abscess", by Zhou Y et al., published in MicrobiologyOpen. 2017;6:e515; https://doi.org/10.1002/mbo3.515 without being referenced. The authors apologize for the mistake.

The correction reads as follows:

\section{Tables}

Table 1 Antimicrobial susceptibility of Pannonibacter phragmitetus 31801 (Table taken from Zhou et al. 2017)

Table 4 Resistance genes on the Pannonibacter phragmitetus 31801 chromosome genome extracted by CARD analysis (Table taken from Zhou et al. 2017)

\section{Figures}

Figure 1 Phyologenetic relationship between Pannonibacter phragmitetus $\mathrm{P}$. phragmitetus 31801 and other closely related species (identity indicated by *) based on $16 \mathrm{~S}$ rRNA nucleotide sequence data. The corresponding GenBank accession numbers are indicated in parentheses. Rhodobacteraceae bacterium SH22-2a was used as an outgroup (Figure taken from Zhou et al. 2017)

Figure 2 Circular representation of the genome of Pannonibacter phragmitetus 31801. Concentric rings, numbered

The original article can be found online at https://doi.org/10.1007/ s00203-018-1522-2.

De-song Ming

drdesongming@hotmail.com

1 Department of Clinical Laboratory, Quanzhou First Hospital Affiliated to Fujian Medical University, Quanzhou 362000, Fujian, China from outer to inner rings, represent the following: coding sequences (CDS; light blue), rRNA genes (pink), tRNA genes (brown), GC content (percentage) as a peak to valley profile (black), GC-skew graph (purple and green). The scale starts at the center circle and runs in a clockwise direction (Figure taken from Zhou et al. 2017)

Figure 3 Subsystem distribution of Pannonibacter phragmitetus 31801 based on RAST annotation server data (Figure taken from Zhou et al. 2017)

\section{Discussion}

In the third paragraph: The genes associated with multidrug resistance efflux pumps could be classified into four groups based on the anti-biotics to which they confer resistance: (1) macrolides: adeJ, cmeB, macAB-TolC, mdtF, mexB, mexD, mexY, smeE, $c f r A$; (2) tetracycline: acrB, adeA, adeB, adeG, adeJ, техB, техY, smeE; (3) fluoroquinolones: $a c r B$, acrF, adeG, adeJ, сеоB, стеB, mdtF, техB, техD, техF, mexI, mexY, smeB, smeE; (4) aminoglycosides: $a c r D, a m r B$, сеоB, mexY, smeB. All these genes encodes individual subunits of the efflux pumps (Zhou et al. 2017).

In the sixth paragraph: In $P$. aeruginosa, mexY promotes aminoglycoside resistance. The proximal binding pocket within MexY, which is jointed with a periplasmlinked cleft, confers the resistance (Lau et al. 2014). Interestingly, the AcrB drug efflux pathway shows a similar conformation. The genome of strain 31801 contained both mexY and $\operatorname{acr} B$, but the isolate remained sensitive to amikacin, highlighting the inconsistencies between AST and CARD database analyses in the present study. Over-expression of $m e x X Y$ in $P$. aeruginosa promoted resistance to aminoglycosides (Sobel et al. 2003), and mexY could be induced by chloramphenicol, tetracycline, macrolides, and aminoglycosides (Jeannot et al. 2005). SmeB and mexB, which showed $52 \%$ identity to each other, were also found in strain 31801 (Zhou et al. 2017). 